Perspective

\title{
Clinical Efficacy of Ovarian Cancer Screening
}

\author{
Masafumi Koshiyama ${ }^{凶}$, Noriomi Matsumura, Ikuo Konishi \\ Department of Gynecology and Obstetrics, Kyoto University Graduate School of Medicine, 54 Shogoin, Kawahara-cho, Sakyo-ku, Kyoto, 606-8507, Japan. \\ $\triangle$ Corresponding author: Masafumi Koshiyama, 54 Shogoin Kawahara-cho, Sakyo-ku, Kyoto, 606-8507, Japan. +81-75-751-3269 (voice), +81-75-761-3967 \\ (facsimile), E-mail: koshiyamam@nifty.com. \\ (C) Ivyspring International Publisher. Reproduction is permitted for personal, noncommercial use, provided that the article is in whole, unmodified, and properly cited. See \\ http://ivyspring.com/terms for terms and conditions.
}

Received: 2015.12.06; Accepted: 2016.03.15; Published: 2016.06.25

\begin{abstract}
Various trials of ovarian cancer screening programs have been reported worldwide. In 2011, one of the most famous papers indicated that annual screening using CA125/transvaginal sonography (TVS) did not reduce ovarian cancer mortality in the United States of America (USA). To investigate the validity of ovarian cancer screening, we verified the analyses of previous reports. At first, we obtained the USA datasets that were used for the analyses and identified many patients in whom cancers were accidentally detected several years after the screening period. We thus performed a new prognostic comparison between the screening group (cancers that were detected through screening or within one year after screening) and the control group (cancers that were found more than one year after screening, without screening, or in the original control group). The results showed that the prognoses of the screening group were significantly better than those of the control group ( $p=0.0017)$. In addition, the screening group contained significantly fewer stage IV cases than the control group $(p=0.005)$. In another screening in the United Kingdom, ovarian cancer was detected at a relatively earlier stage (stage $\mathrm{I} / \mathrm{ll}: 44 \%$ ), while the rate of stage IV detection was low (4\%). Very recently, this team showed significant difference in the rates with and without screening $(p=0.021)$ when prevalent cases were excluded and indicated the delayed effect of screening. These results contrasted with the USA data. In other studies in the USA and Japan, annual screening was also associated with a decreased stage at detection. New histopathological, molecular and genetic studies have recently provided two categories of ovarian carcinogenesis. Type I carcinomas are slow-growing neoplasms that often develop from benign ovarian cysts. Type II carcinomas are high-grade clinically aggressive neoplasms. The rate of type II carcinomas is significantly higher in Europe and the USA than in Asia $(p<0.001)$. Conversely, type I carcinomas are relatively common in Asia. These data theoretically imply that annual screening would be more effective in Asia.
\end{abstract}

Key words: ovarian cancer screening, CA125, transvaginal sonography, Kaplan-Meier survival curve.

\section{Introduction}

Various trials of ovarian cancer screening programs have been reported worldwide. One of these reports was an examination of a screening program for prostate, lung, colorectal and ovarian cancer (PLCO) in the United States of America (USA) that was performed using a randomized controlled trial [1,2] and the largest study for ovarian cancer screening with survival data. The screening strategy involved annual screening with transvaginal sonography (TVS) and CA125 level measurements (CA125 cut-off of $\geqq 35$. Both methods were performed for three years. CA125 levels were measured at years four and five. After that, follow-up was performed for more than five years). The authors' conclusion was that simultaneous screening with measurement of the CA 125 level and TVS did not reduce ovarian cancer mortality compared with the standard care.

To investigate the validity of ovarian cancer screening, we verified the analyses of previous reports. For our analysis, we selected reports throughout the world in which large-scale investigations were performed in population of more 
than 30,000. We herein discuss whether ovarian cancer screening is globally effective.

\section{Original analysis of the PLCO screening data}

The PLCO screening arm involved a randomized controlled trial (RCT) of 78,216 females aged 55 to 74 years assigned to receive either annual screening $(n=39,105)$ or usual care $(n=39,111)$ at 10 centers across the USA between 1993 and 2001 (white, non-Hispanic: 67,401 (86.2\%), Asian: 2,567 (3.3\%)). Ovarian cancer was diagnosed in 212 patients $(0.54 \%)$ in the intervention group and 176 patients $(0.45 \%)$ in the usual care group. The stage distribution in the intervention group was as follows: $32(15 \%)$ cases of stage I disease, $15(7 \%)$ cases of stage II disease, 120 (57\%) cases of stage III disease and $43(20 \%)$ cases of stage IV disease, indicating that the majority of patients had cancer at stage III or above. The distribution of cancer histologies included $116(80 \%)$ cases of serous lesions, five $(3 \%)$ cases of mucinous lesions, $19(13 \%)$ cases of endometrioid lesions and six $(4 \%)$ cases of clear cell lesions, thus indicating that the majority of cases involved serous cancer. We identified a large number of patients whose cancers were accidentally detected several years after the screening period in the intervention group.

We obtained the authors' datasets and performed a new analysis to examine the efficacy of annual screening. We divided the patients who were currently diagnosed with ovarian cancer into two groups to investigate the direct effect of screening. Group A (101 patients) included patients whose ovarian cancers were detected through annual screening (CA125 and/or TVS) or within one year after screening. Group B (344 patients) included patients in the screening group whose ovarian cancers were found over one year after screening due to the patient experiencing symptom, and patients in the no screening or in the control group. We drew Kaplan-Meier survival curves for Group A and Group $B$. The results showed a significantly better prognosis for patients in Group A than those in Group B (median survival; 6.1 vs. 3.3 years, $p=0.0017$ using the log-rank test) (Figure 1). Statistical analyses were performed by two evaluators using the JMP PRO11 software program (SAS Institute Inc). In addition, the former group contained significantly fewer stage IV cases than the latter group ( $13 \%$ vs. $29 \%$, respectively, $p=0.005)$. Moreover the rate of surgery resulting in no macroscopically residual disease was relatively higher in the former group than in the latter group (50,0\% vs.38.0\%, respectively, $p=0.034$ ). In brief, if a patient receives an annual screening examination for ovarian cancer, the stage at detection of advanced cases may shift from stage IV to stage III or below, thus allowing the patients monitored with annual screening to undergo more thorough surgeries than those without screening. Therefore, the patients whose ovarian cancers are found during the annual screening or within one year after the screening may have better prognoses than those who do not undergo annual screening.

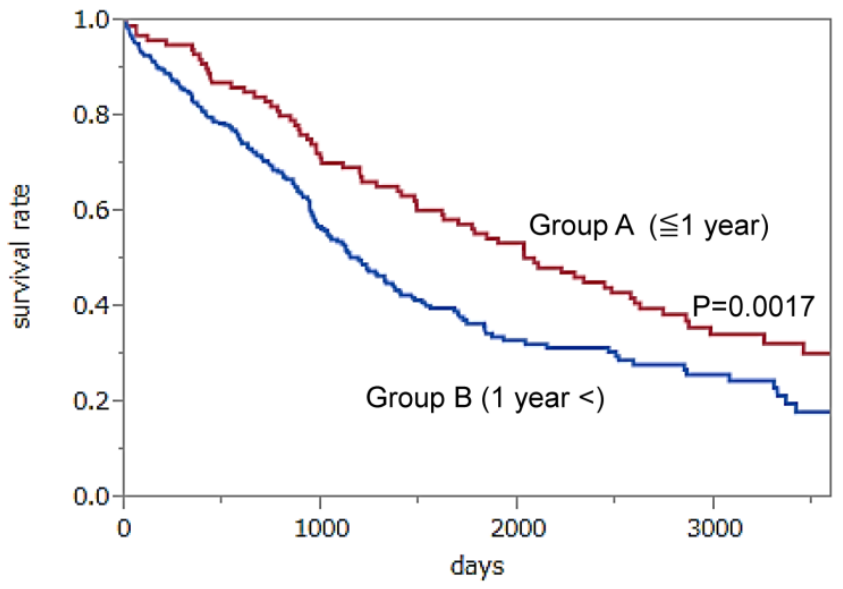

Figure 1. Kaplan-Meier survival curves for Group A (102 patients whose ovarian cancers were detected through annual screening or within one year after screening) and Group B (344 patients whose ovarian cancers were found symptomatically over one year after screening, in the no screening or in the control group). The results showed a significantly better prognosis in Group A than in Group B ( $p=0.0017)$. Hazard Ratio: $0.6235,95 \% 0.4517-0.8794$.

However, we could not definitively determine the efficacy of screening because the survival rates must be compared after randomization with or without screening in order to avoid a "lead time bias" which is caused by the length of time between the detection of a cancer by screening and its usual clinical presentation after the patient's symptoms. Our original study of the PLCO screening data was a retrospective analysis, and was limited by lead time bias, which means that its results must therefore be interpreted with caution. However, the PLCO study was unable to find positive results even in the stage shift and the ratio of complete surgery probably because their methods have various weak points with respect to screening, such as 1) the group undergoing annual screening included women who did not receive screening; and 2) many patients with ovarian cancer in the screening group were diagnosed more than one year after screening. Accordingly, there were many ovarian cancer cases that were thought to have occurred several years after the screening period in the intervention group, which were not related to the direct effect of screening. As mentioned above, doubt has been raised as to whether the content of the screening intervention was sufficient. 


\section{The United Kingdom Collaborative Trial of Ovarian Cancer Screening}

Currently, the largest screening trial is ongoing. The United Kingdom Collaborative Trial of Ovarian Cancer Screening (UKCTOCS) is an RCT of 202,638 women aged 50-74 years (control: 101,359, multimodal screening (MMS): 50,640, TVS alone: 50,639, white $96.5 \%$, Asian 0.9\%) who were assigned between 2001 and 2005 [3-5]. The MMS protocol included annual CA125 screening interpreted using a patented "Risk of Ovarian Cancer" algorithm (ROCA) with TVS as a second-line test [6,7]. Ovarian cancer was diagnosed in $38(0.08 \%)$ patients in the MMS group and $32(0.06 \%)$ patients in the TVS group, including screen negative cases. The distribution of the cancer histologies was similar to that of the PLCO group. The distribution of the cancer stages in the MMS group was as follows: $17(45 \%)$ patients with stage I disease, two (5\%) patients with stage II disease, $19(50 \%)$ patients with stage III disease and zero $(0 \%)$ patients with stage IV disease. The distribution of the cancer stages in the TVS group was as follows: 10 $(31 \%)$ patients with stage I disease, two $(6 \%)$ patients with stage II disease, 10 (53\%) patients with stage III disease and two (9\%) patients with stage IV disease. In total, the stages at detection were relatively earlier (stage I/II: $44 \%$ ), and the rate of stage IV disease was very small $(4 \%)$. For women at high risk of ovarian cancer, the same UK team previously demonstrated a stage shift in the patients who underwent screening within one year compared with the patients who were diagnosed more than one year after the last negative screening ( $\geq$ Stage IIIc; $26.1 \%$ vs $85.7 \%$, respectively, $\mathrm{p}=0.009$ ) [8]. Very recently, the UK team reported the final mortality rate after extending the screening to December 2011 [9]. Follow-up was extended to December 2014. A prespecified analysis of death from ovarian cancer in an MMS cohort versus no screening with the exclusion of prevalent cases showed a significantly different death rate $(p=0.021)$, with an overall average mortality reduction of $20 \%$ (a reduction of $8 \%$ in years $0-7$ and $28 \%$ in years $7-14$ ) in favor of MMS. They insisted that this late effect of screening was predictable in view of the unavoidable interval from randomization to diagnosis and then death. For example, the median interval from randomization to death in the no screening cohort was more than 8 years. The UK team suggested that MMS screening was more effective after 7 years of screening. Thus, the results differed from those of the PLCO screening due to the extension of the screening and follow-up periods.

\section{The Kentucky Screening Study}

In the Kentucky Screening Study, single-arm annual TVS screenings of 37,293 women were performed from 1987 to 2011 [10,11], through which 47 cases of ovarian cancer were detected. Additionally, 12 cases were diagnosed as ovarian cancer within 1 year of a negative screening. We obtained clinical data from the author by personal communication. The stage distribution of the 59 cases in the annual screening group was as follows: 23 (39\%) stage I lesions, 13 (22\%) stage II lesions, 23 $(39 \%)$ stage III lesions, and zero (0\%) stage IV lesions, with a $61 \%$ rate of early stage (I/II) disease. The distribution of cancer histologies included 38\% with serous lesions, $2 \%$ with a mucinous lesion, $26 \%$ cases with endometrioid lesions, $4 \%$ cases with clear cell lesions and $30 \%$ cases with other adenocarcinomas. The survival rate of the patients with ovarian cancer in the annual screening group was $74.8 \% \pm 6.6 \%$ at five years, which compared favorably with the five year survival rate of $53.7 \% \pm 2.3 \%$ among the patients with ovarian cancer who did not undergo screening $(p<0.001)$. The study algorithm indicated that patients began receiving screenings at four- to six- week intervals if there were any abnormal adnexal findings, such as a benign tumor. The authors concluded that annual TVS screening is associated with a decreased stage at detection, as well as a decrease in case-specific ovarian cancer mortality. Furthermore, the rate of stage IV disease was zero. However, that study was not an RCT and demonstrated a significant healthy-volunteer effect, which makes it difficult to accurately interpret the outcomes [12]. Moreover, compared with the PLCO data, the rate of serous cancers was relatively low and the rate of endometrioid cancers was relatively high.

\section{The Japanese study}

In Japan, the results of the Shizuoka Cohort Study of Ovarian Cancer Screening were recently reported. This study was an RCT of 82,487 low-risk postmenopausal women (intervention group: 41,688, control group: 40,799) who were screened using annual TVS and CA125 levels based on a cut-off value [13]. Women with scan findings of benign diseases returned for repeat scans every 3-6 months. The total number of ovarian cancers in the intervention group was $27(0.06 \%)$. The stage distribution in the intervention group was as follows: $17(63 \%)$ cases of stage I disease, one (4\%) case of stage II disease, seven $(26 \%)$ cases of stage III disease and two $(7 \%)$ cases of stage IV disease. The distribution of the cancer histologies included eight $(30 \%)$ cases of serous lesions, four $(15 \%)$ cases of mucinous lesions, five 
(19\%) cases of endometrioid lesions, nine $(33 \%)$ cases of clear cell lesions and one $(4 \%)$ case of another cancer, meaning that the majority of the cases involved histologies other than serous cancer. The proportion of stage I/II ovarian cancers was higher in the screened group $(67 \%)$ than in the control group (44\%), but this difference did not reach statistical significance $(p=0.116)$. The rate of complete surgical excision was higher in the screening group $(21 ; 78 \%)$ than in the control group $(15 ; 47 \%)(p=0.018)$. The mortality rates in that study are unknown.

\section{Two types of ovarian cancer}

New histopathological, molecular and genetic studies have recently provided a better model for ovarian carcinogenesis, including two broad categories, designated as type I, in which precursor lesions in the ovaries have clearly been described, and type II, in which such lesions have not been clearly described and tumors may develop de novo from the tubal and/or ovarian surface epithelium [14]. Type I carcinomas are, in general, slow-growing, indolent neoplasms, and include low-grade serous (rare tumors), mucinous, endometrioid, clear cell and transitional cell cancers. As we previously reported, Type I carcinomas often develop from benign ovarian cysts, and those detected within one year (mean: 7.1 \pm 2.8 months) after the visit for follow-up of ovarian cysts were mostly stage I diseases (17/19; 89\%) [15]. In contrast, type II carcinomas are high-grade clinically aggressive neoplasms, and include a majority of high-grade serous cancers, which are often found at an advanced stage and are highly genetically unstable. The majority of type II carcinomas, but not type II carcinomas, have TP53 mutations, and almost half of the cases involve hypermethylation, or a dysfunction of the breast cancer gene (BRCA 1/2). These biological aspects are evidence of the aggressive nature of type II carcinoma.

\section{Different effects of ovarian cancer screening among different races}

Among different races (Europeans, Asians and subjects in the USA), there are significant differences in the rates of the different histological subtypes of ovarian cancer [16-21]. Figure 2 shows the rates of these histological subtypes of ovarian cancer in Europe, the USA and Asia. The rate of aggressive ovarian cancer, such as high-grade serous cancer (type II), is significantly higher in Europe and the USA than in Asia (Figure 3, p<0.001). Conversely, type I carcinomas, indolent carcinomas arising from precursors, are relatively common in Asia. These data theoretically imply that ovarian cancer screening using CA125/TVS would be more effective in Asia, detecting cancer at an earlier stage or its precursor such as ovarian cyst and thus reducing mortality. In addition, annual screening can be used to detect precursors of type I ovarian cancer, such as cystic tumors, so patients can be closely observed.
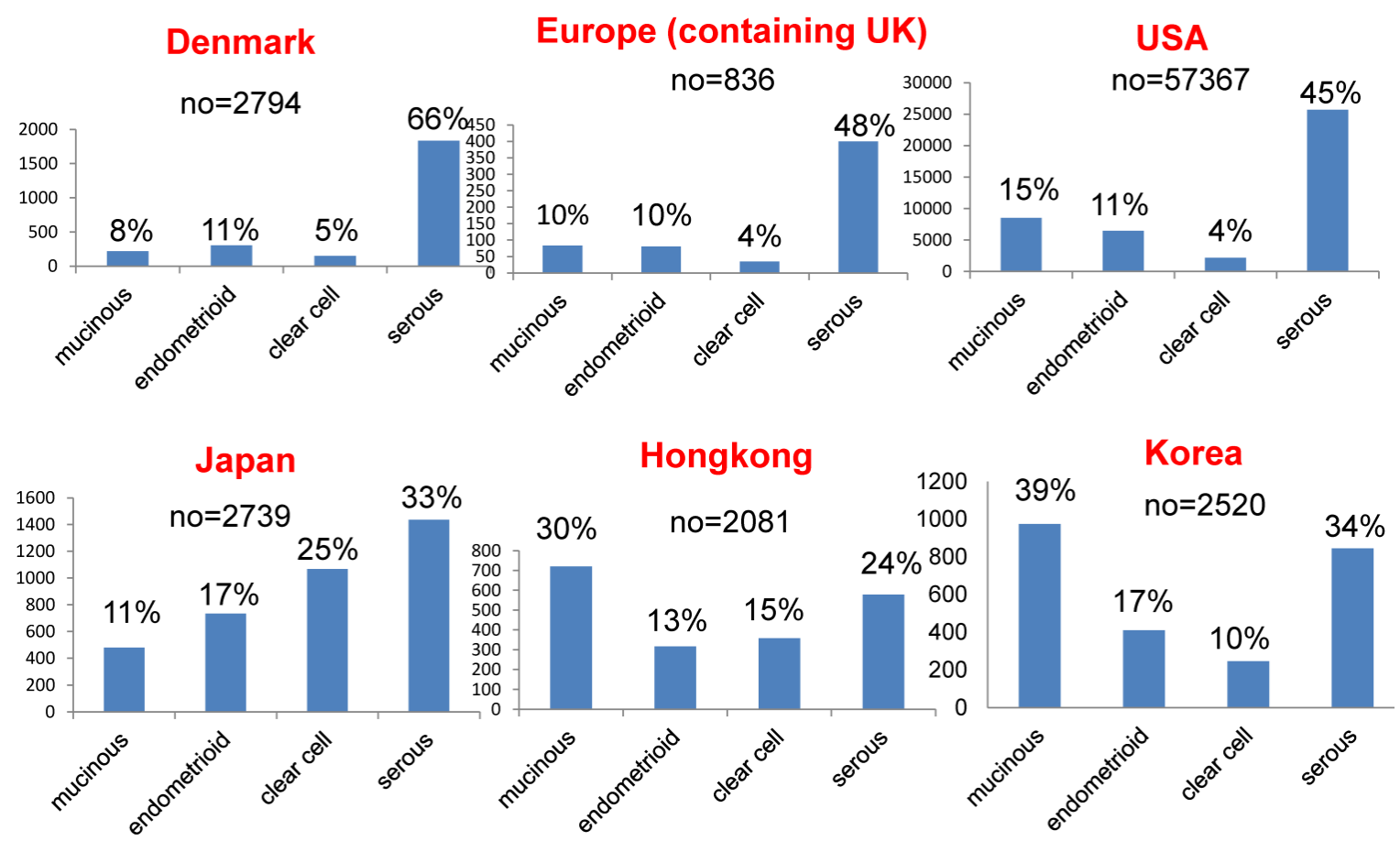

Figure 2. Comparison of the rates of different histological subtypes of ovarian cancer in Europe, the USA and Asia. 
Denmark

no $=2794$

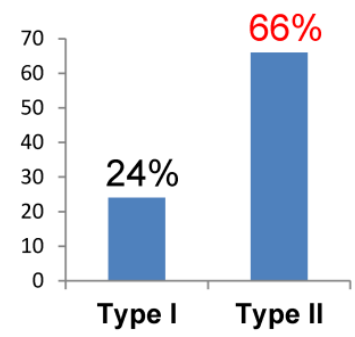

Japan

no $=2739$

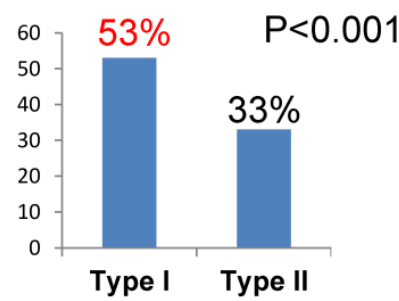

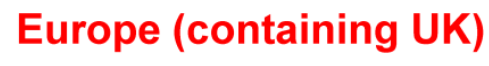

no $=836$

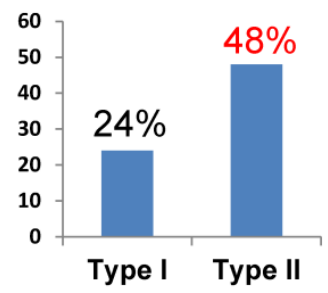

Hongkong

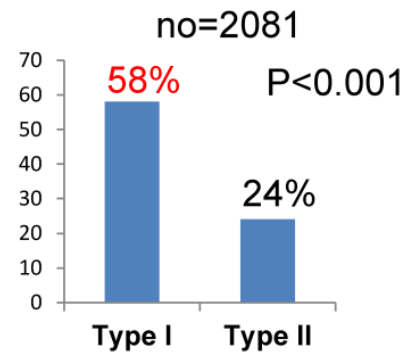

USA

no $=57367$
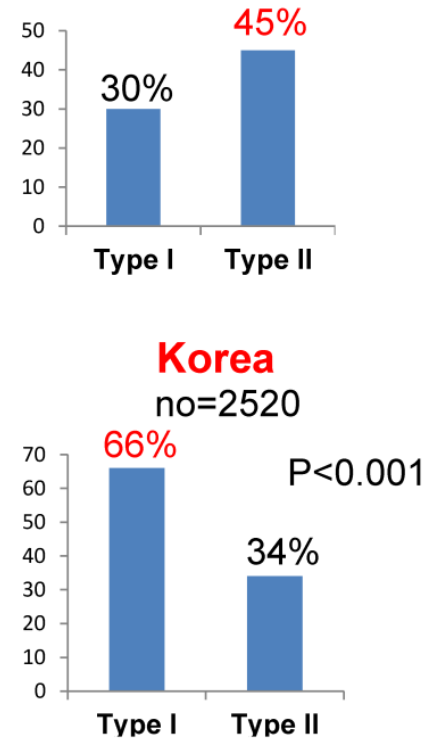

Figure 3. Comparison of the rates of type I and type II ovarian carcinomas among different races. Type II cancer was significantly more common in Europe and the USA than in Asia $(p<0.001)$. Conversely, type I was relatively common in Asia. There were unidentified cancers in addition to type I and type II cancers.

Furthermore, annual screening may improve prognoses, even in cases of type II ovarian carcinoma. The MMS method employed in the UKCTOCS can be used to detect cancer at an earlier stage than the PLCO method. It showed a significant reduction in mortality due to its extended screening and follow-up periods.

\section{Conclusion}

In summary, annual screening with the use of CA125/TVS can detect the precursors of type I ovarian carcinoma, such as cystic tumors, and identify patients who should be closely observed. Thus annual screening may be useful for detecting type I ovarian carcinoma at an earlier stage. Furthermore, annual screening may improve the prognoses or induce the down staging (from stage IV to stage III) of cases of type II ovarian carcinoma, which would further increase its efficacy in Asia, and also make it effective in Europe and the USA, just as the UKCTOCS showed a significant mortality reduction in the UK.

\section{Acknowledgements}

The authors requested the datasets of the PLCO and obtained sources for this article from Dr. Christine D. Berg, who was a corresponding author of the PLCO cancer screening randomized controlled trial. We would like to thank Dr Christine D. Berg and PLCO Project Team who graciously sent the PLCO data to us. The authors thank Dr John Rensselaer van Nagell Jr and coauthors for offering the data of the Kentucky Screening Study. The authors thank the members of the Department of Epidemiology and Healthcare Research, Kyoto University for their helpful assistance with the statistical data analyses.

\section{Competing Interests}

The authors have no financial conflicts of interest relevant to the present work to declare.

\section{References}

1. Buys SS, Partridge E, Greene $\mathrm{MH}$ et al. Ovarian cancer screening in the Prostate, Lung, Colorectal and Ovarian (PLCO) Cancer Screening Trial: findings from the initial screen of a randomized trial. Am J Obstet Gynecol 2005; 193: 1630-9.

2. Buys SS, Patridge E, Black A et al. Effect of screening on ovarian cancer mortality: the Prostate, Lung, Colorectal and ovarian (PLCO) cancer screening randomized controlled trial. JAMA 2011; 305: 2295-303.

3. Menon U, Gentry-Maharaj A, Hallett R et al. Sensitivity and specificity of multimodal and ultrasound screening for ovarian cancer, and stage distribution of detected cancers: results of the prevalence screen of the UK Collaborative Trial of Ovarian Cancer Screening (UKCTOCS). Lancet Oncol 2009; 10: 327-40.

4. Sharma A, Gentr-Maharaj A, Burnell $\mathrm{M}$ et al. Assessing the malignant potential of ovarian inclusion cysts in postmenomausal women within the UK Collaborative Trial of Ovarian Cancer Screening (UKCTOCS): a prospective cohort study. Gynecol Oncol 2012;119: 207-19.

5. Sharma A, Apostolidou S, Burnell M et al. Risk of epithelial ovarian cancer in asymptomatic women with ultrasound-detected ovarian masses: a prospective cohort study within the UK collaborative trial of ovarian cancer screening (UKCTOCS). Ultrasound Obstet Gynecol 2012; 40: 338-44.

6. Skates SJ, Xu FJ, Yu YH et al. Toward an optimal algorithm for ovarian cancer screening with longitudinal tumormarkers. Cancer 1995; 76: 2004-10.

7. Skates SJ. Ovarian cancer screening: develop of the risk of ovarian cancer algorithm (ROCA) and ROCA screening trials. Int J Gynecol Cancer 2012; 22: S24-6.

8. Rosenthal AN, Fraser L, Manchanda R et al. Results of annual screening in phase I of the united Kingdom familial ovarian cancer screening study highlight the need for struct adherence to screening schedule. J Clin Oncol 2013; 31: 49-57.

9. Jacobs IJ, Menon U, Ryan A et al. Ovarian cancer screening and mortality in the UKcollaborative trial of ovarian cancer screening (UKCTOCS): a randomized controlled trial. Lancet. 2015; Dec 17. pii: S0140-6736(15)01224-6: $1-12$. 
10. van Nagell JR Jr, DePriest PD, Ueland FR et al. Ovarian cancer screening with annual tranvaginal sonography. Cancer 2007; 109: 1887-96.

11. van Nagell JR Jr, Miller RW, DeSimone CP et al. Long-term survival of women with epithelial ovarian cancer detected by ultrasonographic screening. Obstet Gynecol 2011; 118: 1212-21.

12. Jacobs I, Menon U. Can ovarian cancer screening save lives? The question remains unanswered. Obstet Gynecol 2011; 118: 1209-11.

13. Kobayashi H, Yamada $Y$, Sato $T$ et al. A randomized study of screening for ovarian cancer: a multicenter study in Japan. Int J Gynecol Cancer 2008; 18: 414-20.

14. Koshiyama M, Matsumura N, Konishi I. Recent concepts of ovarian carcinogenesis: Type I and Type II. (Review) Biomed Res Int 2014; 2014: 934261.

15. Horiuchi A, Itoh $\mathrm{K}$, Shimizu $\mathrm{M}$ et al. Toward understanding the natural history of ovarian carcinoma development: a clinicopathological approach. Gynecol Oncol 2003; 88: 309-17.

16. Sperling C, Noer MC, Christensen IJ et al. Comorbidity is an independent prognosis factor for the survival of ovarian cancer: A Danish register-based cohort study from a clinical database. Gynecol Oncol 2013; 129: 97-102.

17. Gram IT, Lukanova A, Brill I et al. Cigarette smoking and risk of histological subtypes of epithelial ovarian cancer in the EPIC cohort study. Int J Cancer. 2012; 130: 2204-10.

18. Goodman M, Howe HL. Descriptive epidemiology of ovarian cancer in the United State, 1992-1997. Cancer 2003; 97 (10 Suppl): 2615-30.

19. Japan Society of Obstetrics and Gynecology. Statistics of gynecologic tumors in Japan. Acta Obstet et Gynaecol Japonica 2012; 64: 1029-141.

20. Wong KH, Mang OW, Au KH, Law SC. Incidence, mortality, and survival trends of ovarian cancer in Hong Kong, 1997 to 2006: a population-based study. Hong Kong Med J 2012; 18: 466-74.

21. Chung HH, Hwang SY, Jung KW et al. Ovarian cancer incidence and survival in Korea: 1993-2002. Int J Gynecol Cancer 2007; 17: 595-600. 